\title{
New pathological findings in emphysema of childhood: 1. Polyalveolar lobe with emphysema
}

\author{
ALISON HISLOP and LYNNE REID \\ Department of Experimental Pathology, Institute of Diseases of the Chest, \\ Brompton Hospital, London, S.W.3
}

\begin{abstract}
A new pathological entity is here described-a polyalveolar lobe with or without emphysemagiving rise to the clinical features of childhood lobar emphysema.

A detailed and quantitative study of the airways, alveoli and arteries was carried out on the left upper lobe removed because of shortness of breath, thought to be due to "childhood lobar emphysema'. The child was 17 days old and the radiograph showed hypertransradiancy of the left lung. The alveolar number was increased five-fold. Alveolar size was normal, so it was found that emphysema, accepted today as a structural diagnosis, was not present. The increase in alveolar number seemed confined to the apical, posterior, and anterior segments, the lingula being unaffected. By contrast, the airways and arteries were normal for age in number, size and structure, suggesting that the condition was a 'giantism' of the alveolar region. The blood flow was probably too low for the lobar volume; certainly the excessive alveolar number could not be due to increase in blood flow.

In two further specimens, previously dissected, a similar polyalveolar condition was found, associated with emphysema. The patients were older at the time of surgical resection and the emphysema may have developed post-natally. In all three cases the radiographic features had suggested emphysema. It is suggested that the condition be called 'polyalveolar lobe', 'with emphysema' or 'without emphysema' being added as a separate item to the description.
\end{abstract}

Infantile lobar emphysema (also called congenital lobar emphysema and congenital obstructive emphysema) usually presents clinically in early childhood because of acute dyspnoea. The radiological appearance is of a hypertransradiant lobe with compression of the remainder of the lung and contralateral displacement of the heart.

It has been suggested that the condition arises from (1) infection damaging alveoli (Leape and Longino, 1964), (2) developmental abnormality of alveoli (Bolande, Schneider, and Boggs, 1956), (3) an obstruction to the lobar bronchus perhaps from inspissated secretion (Thomson and Forfar, 1958), or (4) from abnormal collapsibility of the airway due to absence of cartilage (Bolande et al., 1956) or abnormality of cartilage (Stovin, 1959).

It has recently been emphasized that clinical features of infantile lobar emphysema may arise from several different pathological entities (Reid, 1967a). For example, atresia of the apico-posterior bronchus of the left upper lobe, as reported in the adult (Simon and Reid, 1963; Waddell, Simon, and Reid, 1965), has also been seen by one of us as the cause of this clinical presentation in the child.

The purpose of this paper is to report in detail on the operation specimen from a patient presenting clinically as infantile lobar emphysema, and to refer to two additional cases, all arising from a lung abnormality not previously described-a localized gross increase in alveolar number representing virtually ' giantism' of the alveolar region. The term we suggest to describe this condition is 'polyalveolar lung', with or without emphysema. This finding is based on a detailed study of the airways, arteries and alveoli. Methods of examination not previously applied in this condition have been used, and the way in which these have been combined to give the maximum amount of information is demonstrated.

\section{MATERIAL AND METHODS}

Study in detail of the left upper lobe from a patient with congenital lobar emphysema (case 1) stimulated re-examination of two other left upper lobe specimens, 
though in less detail, since they had already been fixed and cut (cases 2 and 3).

Planimetry was used on the antero-posterior and lateral radiographs of case 1 to assess the increase in lobar volume in life by comparison with the expected volume of the lobe.

Analysis of the airways and arteries demands some idea of their three-dimensional branching pattern and of their size and structures. Methods of alveolar quantification after lung sampling, based on the methods of Chalkley (1943) and recently popularized by Weibel and Gomez (1962) and Dunnill (1962a), are complementary to this analysis.

In the first specimen examined the artery was injected by hand, with a Micropaque gelatin suspension, according to the method of Elliott (see Reid, 1967a). The lung was then inflated and fixed by intrabronchial injection of buffered formol saline. The specimen was radiographed, and the arteriogram revealed the overall pattern of arterial branching and could be used to measure lumen size both at the hilum and progressively along its course (Millard, 1965 ; Davies, 1969).

The volume of the freshly inflated specimen was recorded and also the volume after fixation and before slicing. This specimen was fixed for six months before it was sliced, although one week's fixation suffices. For quantitation the lung is usually sliced through its largest plane, i.e., roughly parallel to the mediastinal surface. On this occasion the specimen was sliced into $1.0-\mathrm{cm}$. slices in a transverse plane so that the axial artery and airway of the posterior segment passed roughly at right angles to the plane of section, which facilitated taking blocks throughout the length of these structures. Random selection of blocks was made but additional blocks were taken so that the whole of the axial pathway from the posterior segment of the upper lobe and tissue from each of the other segments was included.

Before any blocks were taken, macroscopic quantification of the following lung structures was made by a point-counting technique (Dunnill, 1962a): (1) 'alveolar region' (that is, that containing no structure over $1 \mathrm{~mm}$. in diameter); (2) arteries ; (3) bronchi ; (4) pleura and septa.

The airways were traced and, in addition to the number of their branches, the distal extent of cartilage along axial airways was established. If the specimen is large enough, certain airways can be traced by macroscopic dissection to within the last few branches; for these, microscopic sections are necessary. This gave the proportion of the total airways that were bronchi, i.e., proximal to the most distal plate of cartilage, and bronchioli, i.e., distal to the ultimate cartilage. The staining characteristics of cartilage were also checked to see, in particular, whether acid glycoprotein had formed.

The arterial branching pattern was reconstructed by using step or serial sections. Wall structure and thickness and external diameter of the arteries were measured over a range of arterial size. These results could be analysed to give the population distribution of small arteries by structure (Elliott, 1964) and the extent of muscle in the arterial tree, estimated both by reference to external diameter and to the airway accompanying the given artery.

The percentage of the 'alveolar region' (see macroscopic point-counting above) that is made up of alveolar air is determined by microscopic pointcounting of the randomly taken lung sections. The number of alveoli and arteries per unit area were also counted. From the analysis of these results the total alveolar number was estimated, the alveoli per millilitre (Davies, 1969; Davies and Reid, 1970) and the alveoli/blood vessel ratio.

Emery and Mithal (1960) described a 'radial alveolar count'. This figure represents the number of alveoli transected by a perpendicular from the respiratory bronchiolus to the perimeter of the acinus, as indicated by 'the nearest and definite connective tissue septum'.

In summary, in addition to a general macroscopic and microscopic examination, the use of the above techniques, in combination, allowed quantification of the following features of lung structure:

\section{ARTERIES}

FROM THE ARTERIOGRAM Arterial lumen diameter at the hilum and its reduction to the periphery

FROM MICROSCOPIC EXAMINATION Number of branches along an axial arterial pathway

Arterial wall thickness related to external diameter Distribution of the types of small pulmonary artery-an arterial 'population count'

Arterial level to which muscle penetrates, established $(a)$ by arterial diameter, and $(b)$ by the structure of the accompanying airway, i.e., terminal or respiratory bronchiolus

BRONCHI

FROM MACROSCOPIC DISSECTION Number of bronchial branches from the axial pathway

FROM MICROSCOPIC EXAMINATION Proportion of bronchi to bronchioli

Diameter of airways

Distal extent of cartilage assessed by airway generation

\section{ALVEOLI}

FROM COMBINED MACROSCOPIC AND MICROSCOPIC POINTcounting Proportion of alveolar and largestructure regions

FROM MICROSCOPIC EXAMINATION Total alveolar number

Number of alveoli/millilitre

Alveoli/blood vessel ratio

Radial alveolar count 
RESULTS

\section{CASE 1}

Case 1 was a male child born at term after a normal pregnancy and weighing $7 \cdot 5 \mathrm{lb}$. $(3.4 \mathrm{~kg}$.) at birth. The immediate neonatal period was normal, but at seven days he became short of breath with marked sternocostal recession and central cyanosis. There was reduced air entry over the left hemithorax; some crepitations were heard over the right lung. No other abnormalities were detected and, in particular, there was no evidence of heart failure.

The chest radiograph (Fig. 1) showed the left lung to be enlarged with downward displacement of the left hemi-diaphragm and deviation of the heart and trachea to the right. The left hemithorax was hypertransradiant; hilar and proximal lung vessels were seen with a virtually normal arrangement.

A provisional diagnosis of severe infantile lobar emphysema was made. There was gradual deterioration in the baby's condition, and at 17 days a left lateral thoracotomy was performed through the 6th interspace. A 'hyper-inflated' upper lobe occupied almost the whole of the hemithorax, but the surgeon commented that the lingula appeared normal. The left lower lobe was aerated and of normal appearance save that it was small. The left upper lobe and lingula were removed and, although the lower lobe expanded satisfactorily, it did not completely fill the left hemithorax. The left lower lobe has gradually expanded to fill the left chest and for 12 months since the child has remained well.

MEASUREMENTS FROM CHEST RADIOGRAPH In the apico-posterior view the area occupied by the left upper lobe was roughly $46 \mathrm{~cm}^{2}$; as the expected area in this child's chest would be only $15 \mathrm{~cm} .^{2}$, this represented a three-fold increase. The left lower lobe was airless. The right lung had an area of only $11 \mathrm{~cm}^{2}$, due to the shift of the heart to this side, although normally in this child it should be about $15 \mathrm{~cm} .^{2}$. In the lateral view the area of the upper lobe was approximately $60 \mathrm{~cm} .^{2}$, compared with a probable normal of $20 \mathrm{~cm}^{2}$-again a three-fold increase.

This three-fold increase in area represents a $3_{2}^{3}$ change in volume; that is an increase in volume of the order of five times.

MACROSCOPIC EXAMINATION AND ARTERIAL INJECTION OF SPECIMEN When received at the laboratory the lobe was mostly aerated, although the lingula was represented by a collapsed region below the hilum with a torn tag of tissue attached. The specimen showed no evidence of obstruction to the airways. The left upper lobe was roughly $8 \times 5.5 \mathrm{~cm}$. in area by $2.5 \mathrm{~cm}$. thick. The arteries to the apical and posterior segments were injected

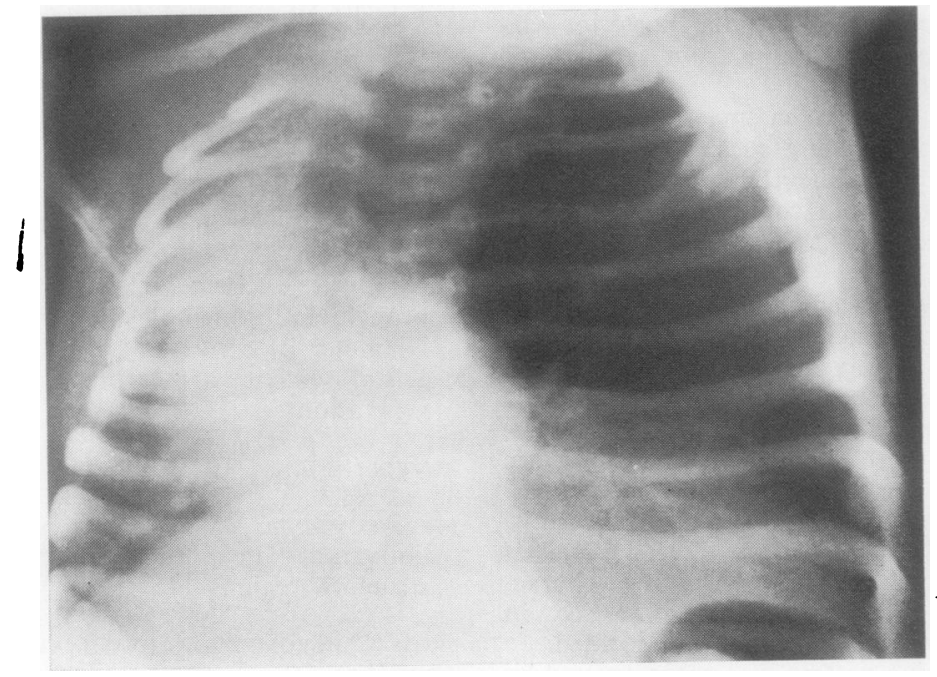

FIG. 1. Case 1. Pre-operative radiograph, showing enlarged, transradiant, avascular left upper lobe. The left hemi-diaphragm is flattened and the heart and trachea are displaced to the right. 
by hand with Micropaque gelatin solution (Fig. 2); the lingular region could not be inflated nor injected because of tearing and of the diffculty in identifying its major structures.

After inflation the volume of the left upper lobe was $125 \mathrm{ml}$. Some months after fixation, when the specimen was studied, the volume was again measured and found to have fallen to $90 \mathrm{ml}$. the normal at this age being about $30 \mathrm{ml}$. Through the pleura the lobular arrangement seemed clear over most of the lobar surface, but there was little evidence of emphysema and this was confirmed when the lobe was cut, there being little retraction below the cut surface.

Macroscopic point-counting of the sliced lung showed that the 'alveolar region' as defined above was $85 \%$ of the total lobar volume, within a normal range.

Alveolar region Microscopically the alveolar walls appeared normal in thickness and contained a normal amount of collagen and reticulin.

The estimated total alveolar number in the lobe was $27.259 \times 10^{6}$, the normal value for the age being $5 \times 10^{6}$ (Dunnill, 1962b). This represented a five-fold increase (see Table).

\section{T A B L E}

ALVEOLAR RESULTS IN THREE CASES OF CHILDHOOD LOBAR EMPHYSEMA (POLYALVEOLAR EMPHYSEMA)

\begin{tabular}{|c|c|c|c|c|c|}
\hline Case & Age & $\begin{array}{l}\text { Lobar } \\
\text { Volume } \\
\text { (fixed) } \\
\text { (ml.) }\end{array}$ & $\begin{array}{c}\text { Total } \\
\text { Alveolar } \\
\text { No. } \\
\left(\times 10^{\circ}\right)\end{array}$ & $\begin{array}{c}\text { Alveoli/ } \\
\text { ml. } \\
\left(\times 10^{\circ}\right)\end{array}$ & $\begin{array}{c}\text { Mean } \\
\text { Radial } \\
\text { Acinar } \\
\text { Alveolar } \\
\text { Wall } \\
\text { Count }\end{array}$ \\
\hline $\begin{array}{l}1 \\
2 \\
3\end{array}$ & $\begin{array}{c}17 / 7 \\
3 / 12 \\
8 / 12\end{array}$ & $\begin{array}{c}90 \\
(30) \\
75 \\
(50)\end{array}$ & $\begin{array}{l}27 \cdot 259 \\
(5)^{1} \\
27 \cdot 65 \\
(18)^{1}\end{array}$ & $\begin{array}{c}0.356 \\
(0.336) \\
0.512 \\
(0.785)^{2} \\
0.377 \\
(0.785)^{2}\end{array}$ & $\begin{array}{l}9.25 \\
(5.5) \\
10.16 \\
(5.5) \\
10 \cdot 16 \\
(6.6)\end{array}$ \\
\hline
\end{tabular}

The normal value at the appropriate age is shown in parentheses 1 Dunnill (1962b)

2 Davies \& Reid (1970)

The number of alveoli per millilitre of fixed alveolar tissue was $0.356 \times 10^{6}$. Comparable figures for a normal lung of this age are not available; for a two-day-old lung it has been estimated to be $0.336 \times 10^{6}$, for a four-month lung $0.785 \times 10^{6}$. This rapid decrease in alveolar size doubtless reflects the great increase in alveolar number just before the eighth week (Boyden and Tompsett, 1965). Boyden has emphasized that before this time the alveolar region is represented by air spaces larger and more simple than adult type alveoli, which he has called 'primitive saccules'. Even at 17 days these are still the main type of airspace in the alveolar region, which suggests that in the present specimen when fixed there was no evidence of emphysema, that is, of alveolar air spaces too large for age. While lung volume during life must be taken into account before denying the presence of clinical emphysema, it can be said that emphysema was not apparent after inflation at the pressures usual in our laboratory.

The radial acinar alveolar count confirmed that there was an increase in the number of alveoli within an acinus. In the apical, posterior and anterior segments (henceforth referred to as the upper three segments) a mean number of 9.25 alveoli lay between the respiratory bronchiolus and the pleura, the normal number for this age being $5 \cdot 5$. In the lingula, on the other hand, the count was within the normal range. It would seem that it is only in the upper three segments that there is an excess of alveoli.

In the upper three segments the number of alveoli was also increased in relation to the number of arteries per unit area of lung. The mean ratio of alveolar/artery number in a microscopic field was 21.8 in the diseased lobe, and is 8.8 in the normal. The number of arteries per square millimetre was also reduced, there being 5.35 in the normal and 2.85 in the abnormal diseased segments. The arteries were reduced per unit number of alveoli and per unit volume of lung. In other respects the arterial system was normal, emphasizing that it is the alveolar increase that is abnormal, that is, the 'emphysematous' left upper lobe showed a five-fold increase in the number of alveoli, probably localized to the three upper segments. The specimen did not show any increase in alveolar size.

Bronchi Reconstruction from serial sections of the axial pathway of the posterior segment of the upper lobe showed that the bronchial branching pattern was normal as was the size of bronchi. The number of generations between the segmental hilum and terminal bronchiolus was 16 , within the normal range, and cartilage extended to between the 7 th and 8 th generations; therefore the proportion of bronchi to bronchioli was also normal.

The cartilage plates in the posterior segment were thin and elongated; in the anterior and lingular segments they appeared normal. Alcian blue staining of the cartilage plates showed that acid glycoprotein was present.

The cartilage plates were normal in number although in the posterior segment they appeared sparser because of the 'stretching' of the airway. Individual plates in this region were thinner but 


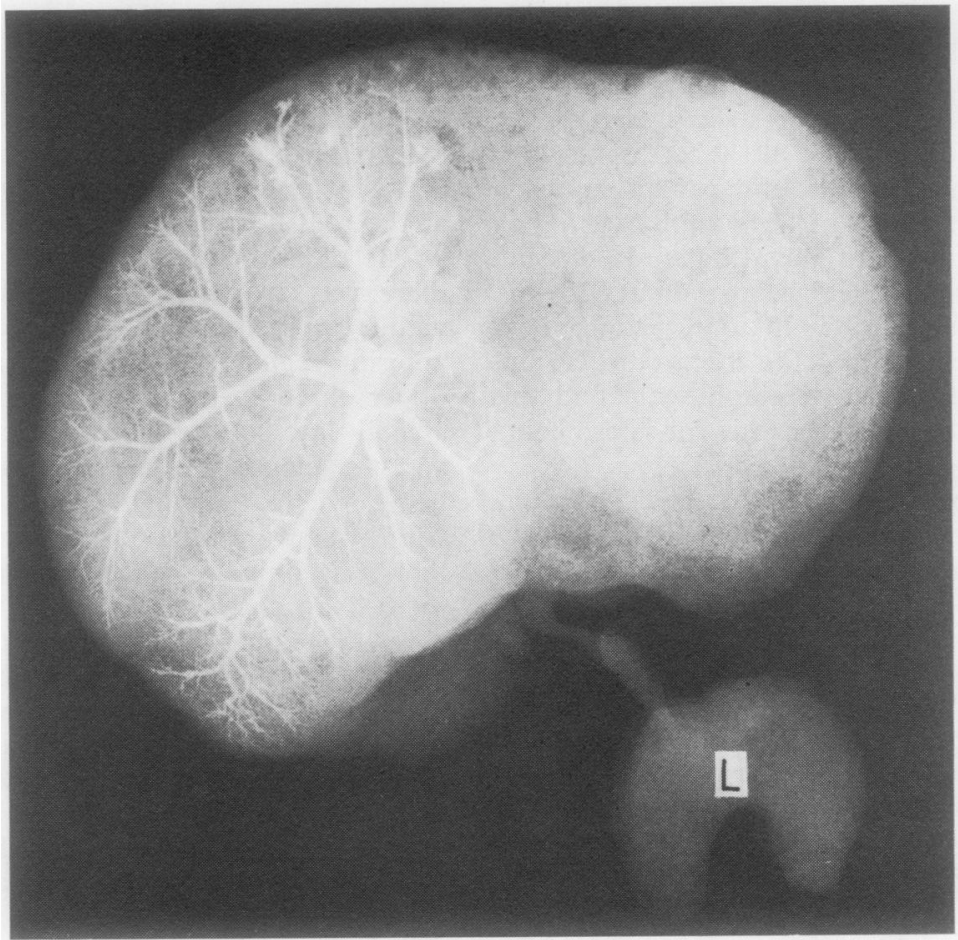

FIG. 2. Case 1. Arteriogram of left upper lobe. The posterior segment is injected with a radio-opaque medium. The arteries appear sparse because of the large size of the lobe. Part of the lingula $(L)$ can be seen as a tag of tissue. $(\times 1$.

covered a larger area of bronchial wall-perhaps because of compensatory overgrowth. It seems that the bronchi were not affected except that the length between branches was increased because of the excessive lobar volume.

It would thus seem that even in the segments with too many alveoli the bronchial tree was normal, suggesting that it was not involved in the disturbance to development. Clustered at the end of the pathways studied, the normal number of terminal bronchioli was found. This, together with the normal branching pattern, suggests that the total number of terminal bronchioli in the lobe was normal, that is, the acinar number was normal. This means that each acinus contained too many alveoli.

Arteries In the arteriogram (Fig. 2) the arteries appeared sparse because of the increase in the volume of the lobe. Lumen diameter along the axial pulmonary artery of the posterior segment was measured at levels from the hilum to the periphery. The diameter at each level was normal for the child's age, but since there was an increase in lobar volume it was small in proportion to the lung it supplied.

The arterial branching pattern along the axial pathway of the posterior segment, when traced microscopically, was found to be normal both in number of branches and in the proportion of supernumerary to conventional type arteries (Elliott, 1964 ; Elliott and Reid, 1965). It might be expected that the increase in alveolar number within an acinus would be associated with an increase in arterial blood supply, but there was no increase in the supernumeraries which supply the acinar periphery, nor was there any increase in blood flow to the acinus since there was no increase in the size of the arterial lumen.

The wall structure of the $1,500 \mu \mathrm{m}$. segmental artery was normal - that of a muscular artery : an elastic arterial media has not been found below $1,700 \mu \mathrm{m}$. at this age (Hislop, 1970).

Random sections showed that throughout the lobe muscle penetrated to a normal level whether judged by the size of the artery or by the structure 
of the airway it accompanied. At this age some wholly muscular arteries are found with terminal bronchioli, but not beyond. The size above which all arteries are muscular is $150 \mu \mathrm{m}$. It thus followed that the external diameter of arteries running with terminal and respiratory bronchioli was normal.

'The distribution by size, or 'population count', of the three types of small artery-muscular, partially muscular and non-muscular-was normal. For arteries below $200 \mu \mathrm{m}$. external diameter the wall thickness of the arteries related to external diameter was normal. For larger sizes the mean wall thickness was slightly increased. This is of doubtful significance since the injection was done by hand so that distension may have been less complete than usual, and other signs of increased muscularity such as extension of muscle to the periphery were absent.

No cross-filling from the pulmonary artery to the bronchial arteries was seen, though in the normal lung at this age with our technique there is a considerable cross-filling. This evidently reduces with age since, after early childhood, none is seen: we have seen considerable cross-filling at four months but not at 18 months or older. Though not injected, it appeared that the veins were normal. There was no arterio-venous crossfilling.
The pulmonary arterial circulation was thus relatively normal in structure. The branching pattern and diameter were normal, suggesting that flow and pressure were normal for age. The former was reduced when related to alveolar number and lobar volume, and there were fewer arteries per alveolus in the intra-acinar region. It can be presumed, therefore, that the blood flow per unit volume of lung was less than in the normal.

The striking abnormality in this case was a fivefold increase in lobar volume associated with a similar increase in alveolar number, but with virtually no increase in alveolar size, and therefore no emphysema. The airways were normal and so were the arteries for age, although this means that the arteries were too few and too small in relation to acinar volume and alveolar number.

In two cases (one described as case 10 in Reid (1967a)) further quantitative examination of the material still available from specimens was made.

\section{CASE 2}

From one patient (female) the left upper lobe had been removed at the age of 3 months, after several weeks of shortness of breath. At operation the lobe was found to be large, the apical segments being most affected (Fig. 3); even

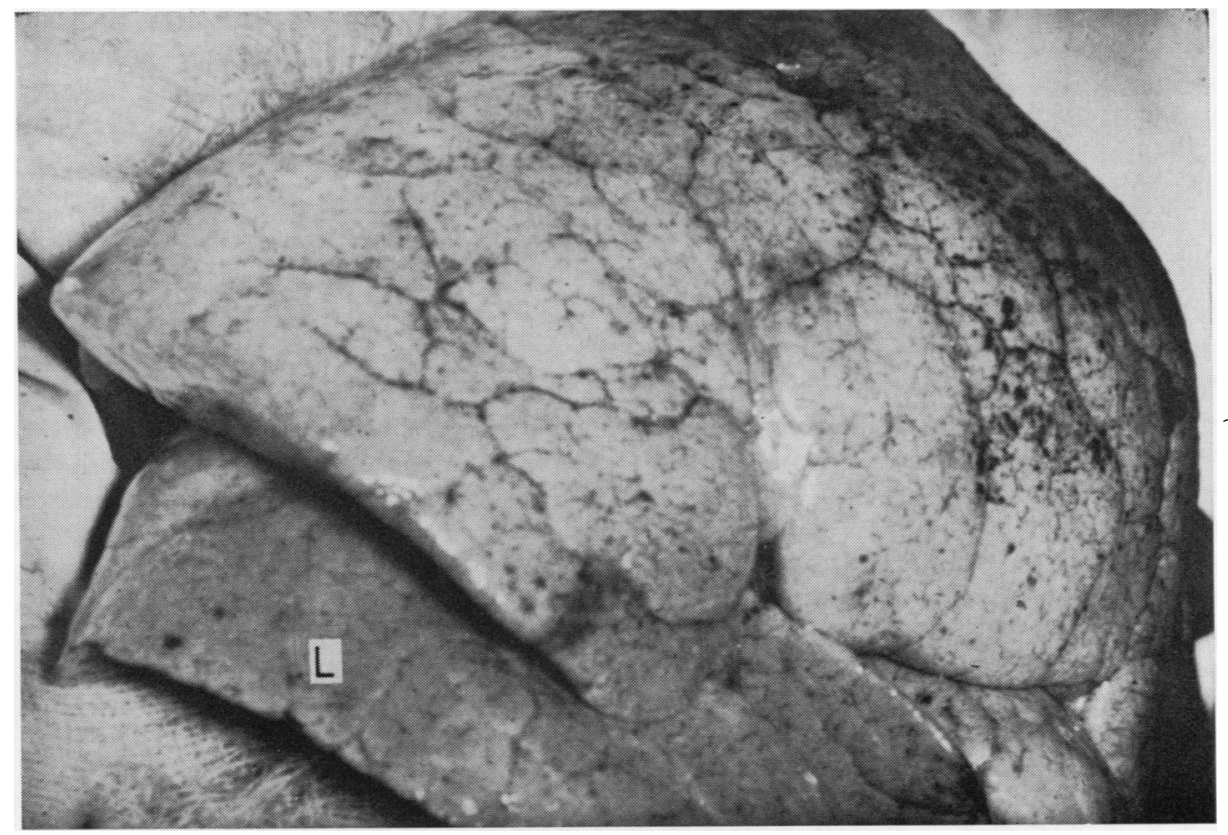

FIG. 3. Case 2. The left upper lobe shows that the apical segments are more inflated than the lingula $(L)$, to produce a groove mimicking the appearance of a fissure. 
after removal from the body this region remained large and showed air trapping, while the lingular and posterior segments collapsed normally. Here also the number of airway generations was normal, when the airway was dissected macroscopically. The cartilage in the anterior and apical segments showed thin prolongations, as described by Stovin (1959), while elsewhere the plates of cartilage appeared normal.

Although the lobar volume is smaller than in case 1 , it was doubtless considerably larger at the time of resection since blocks had been cut from it, thereby causing deflation. By quantitation on further blocks considerable increase in alveolar number could still be shown-27.65 $\times 10^{6}$ instead of the normal $18 \times 10^{6}$ for this age (see Table). The methods of calculation are based on total lung volume which largely corrects for deflation, but not for the tissue removed earlier. The radial acinar alveolar count confirmed an increase in alveolar number since in this case it was $10 \cdot 16$, the normal being $5 \cdot 5$. Some degree of emphysema is present, the number of alveoli per millilitre being $0.512 \times 10^{6}$; the normal would be $0.785 \times 10^{6}$. It would seem, however, that excessive alveoli in a localized region within the lobe is the main abnormality.

\section{CASE 3}

In the third case the left upper lobe was removed from a male child aged 8 months, in whom shortness of breath of moderate severity had been the presenting symptom. In this specimen also, deflation since resection and block taking had affected precise estimation of the alveolar number. The method of radial acinar alveolar count showed that, within the acinus, there was considerable increase of alveoli, the mean number being $10 \cdot 16$ instead of the normal 6.6 at this age. In addition to the increased total alveolar number, slight emphysema was found$0.377 \times 10^{6} \quad$ alveoli $/ \mathrm{ml}$., the normal being $0.785 \times 10^{6}$ alveoli $/ \mathrm{ml}$. (see Table).

It seems then that, although clinically and radiologically the cases were typical of childhood lobar emphysema, the main abnormality is excessive multiplication of alveoli, a condition not previously reported. In case 1 one region of the lobe was affected, and in the other two also it is probable that it was localized. In case 1 firm evidence of emphysema could not be established from examination of the specimen. In cases 2 and 3 emphysema was shown even in the fixed specimen. It is possible that this had developed in the postnatal months.
DISCUSSION

The detailed methods for assessing pulmonary growth described here make it possible to analyse its nature and to suggest the time of onset of any abnormality.

From the Laws of Lung Development (Reid, 1967b) the following general conclusions follow. Reduction in the number of airways below normal points to abnormal growth in the first half of intra-uterine life. A total alveolar number less than the figure found at birth also indicates antenatal disturbance, though probably in the later months; a figure between the normal at birth and that appropriate to a given age suggests postnatal and, perhaps, antenatal slowing. The pre-acinar arterial development is normally in line with bronchial development, the intra-acinar with alveolar (Hislop, 1970). They are not interdependent, however, as is shown by case 1 reported here, where the arteries are normal for age but the alveoli greatly exceed the normal.

In case 1 the airways and pre-acinar arteries were normal, suggesting that development during the first half of intra-uterine life was not disturbed. The lobe was removed so soon after birth that the overgrowth could not have occurred in these few days-particularly as in the normal lung alveoli do not multiply in the first post-natal weeks (Boyden, 1965; Boyden and Tompsett, 1965). It is likely then that the abnormal development started in late fetal life after the canalicular stage.

\section{POLYALVEOLAR LUNG}

The abnormality is in the alveolar number, an estimated five-fold increase for the whole lobe, probably a nine-fold increase for the affected upper three segments. The excess alveoli do not form a separate mass, but since the number of terminal bronchioli is normal they are evenly distributed throughout the acini of the affected region and in normal communication with airways. The lobe is thus 'polyalveolar' with each acinus a 'giant'. In spite of this, all methods showed that the intra-acinar arterial development was normal for the age of the lung but not for its size. This is an instance where blood supply has not followed demand. It suggests that before birth respiratory and circulatory development are independent of each other. 'Polyalveolar lung' would thus seem the best term to describe this condition. 


\section{EMPHYSEMA}

The emphysema embodied in the term 'childhood lobar emphysema' must be separately assessed; its presence may be established from the specimen, and perhaps from the radiograph during life.

In case 1 the radiograph showed transradiancy, avascularity and an increased volume-all signs of air trapping. In general terms, transradiancy arises from a change in air/tissue ratio. The alveolar walls were of normal thickness and there was no significant increase in alveolar size. A reduction in blood increases transradiancy (Reid, 1967a ; Reid, Simon, Zorab, and Seidelin, 1967) and the transradiancy in case 1 probably reflects reduction in blood flow per unit volume of lung. The number of blood vessels per unit volume of lung was reduced, i.e., the amount of blood in the alveolar wall.

The evidence of air trapping was the depression of the left hemi-diaphragm, the bow chest and the displacement of the heart and trachea. The reason for the air trapping is not clear; there may have been premature collapse of the airways or the volume increase with shift of mediastinal structures may have caused kinking of the left main or upper lobar bronchus. Air trapping is a condition usually associated with emphysema, but without further evidence we cannot equate the two because the radiographic features are not truly diagnostic. Emphysema was demonstrated pathologically in cases 2 and 3 even in the fixed specimen, and would certainly have been more severe during life. In these two cases the large air spaces may have been due to interference with alveolar multiplication after birth (Reid, 1967b, 1970). In the specimen of case 1 emphysema was not demonstrated but might have developed if the lobe had not been removed. Shortness of breath and air trapping are sufficient clinical evidence for the diagnosis of emphysema and it would certainly seem justified, perhaps even in case 1 , to describe the clinical condition as a 'polyalveolar lung probably with emphysema'. Certainty of the diagnosis of emphysema must rest on pathological examination.

\section{PATHOGENESIS}

The excessive number of alveoli suggests a 'giantism' or hypertrophy of the affected part of the lung. It is reminiscent of the enlarged limb sometimes associated with a systemic arteriovenous fistula, and possibly brought about by an excessive blood flow. In the present case, however, the diameter of the lobar artery, its pattern of branching and its reduction in size toward the periphery were all normal, suggesting that there had been no significant increase in flow. No crossfilling occurred between the pulmonary and bronchial arteries. Within the alveolar region fewer arteries per alveolus, or per unit of lung area were seen. This points to a reduced flow per unit volume of tissue: certainly it would not seem possible to suggest an increased blood flow as the cause of the condition.

The fact that only part of the lobe seems to be affected has also to be considered. This condition has long been called lobar emphysema, and, certainly to the surgeon at operation and usually to the pathologist in the laboratory, it seems that the whole lobe is affected. There is evidence (Reid, 1967a) that this is certainly not always the case and that, even when the whole lobe appears uniformly increased in volume, different parts of the lobe may behave differently or have suffered unequally. For example, on removal from the body the lung may deflate unevenly, one part of the lobe staying over-inflated while the rest deflates and behaves as normal lung might. The results described here for case 1 confirm that the abnormality is certainly worse in certain segments and may even be confined to one region only. It has not been possible to ascertain whether these changes are precisely localized to a given segment, or to one region independently of segmental arrangement.

It is of interest that in all three cases described here it seemed that the apical three segments were all affected, but the fact that the cartilage more closely resembled normal in the anterior segment could mean that even the apical segments were not affected evenly.

This abnormality of the newborn lung thus represents a localized overgrowth of the alveolar region. The fact that the alveoli are normally differentiated and in normal communication with airways probably distinguishes this condition from hamartoma, in which anatomical derangement is more severe.

The growth achieved, whether in an organ or a part of it, represents a balance between stimulus and inhibition to growth. A local over-action of 'growth' organizer substance could be postulated as the cause of the change described here. In the present state of our knowledge it would not seem possible to go further.

We thank Mr. Charles Drew for allowing us to study the specimens from cases 1 and 3, and $M r$. Arthur Makey for case 2. To Dr. George Simon we are indebted for his opinion on the radiographs. 


\section{REFERENCES}

Bolande, R. B., Schneider, A. F., and Boggs, J. D. (1956). Infantile lobar emphysema; an etiological concept. Arch. Path., 61, 289.

Boyden, E. A. (1965). The terminal air sacs and their blood supply in a 37-day infant lung. Amer. J. Anat., 116, 413.

— and Tompsett, D. H. (1965). The changing patterns in the developing lungs of infants. Acta anat. (Basel), 61, 164.

Chalkley, H. W. (1943). Method for the quantitative morphologic analysis of tissues. $J$. nat. Cancer Inst., 4, 47.

Davies, G. (1969). The effect of chronic lung disease in childhood on lung growth, including measurement of right ventricular weight, alveolar development and pulmonary artery structure. M.D. thesis, Cambridge.

- and Reid, Lynne (1970). Growth of the alveoli and pulmonary arteries in childhood. Thorax, 25, 669.

Dunnill, M. S. (1962a). Quantitative methods in the study of pulmonary pathology. Thorax, 17, 320 .

- (1962b). Postnatal growth of the lung. Thorax, 17, 329.

Elliott, F. M. (1964). The pulmonary artery system in normal and diseased lungs-structure in relation to pattern of branching. Ph.D. thesis, University of London.

— and Reid, L. M. (1965). Some new facts about the pulmonary artery and its branching pattern. Clin. Radiol., 16, 193.

Emery, J. L., and Mithal, A. (1960). The number of alveoli in the terminal respiratory unit of man during late intrauterine life and childhood. Arch. Dis. Childh., 35, 544.
Hislop, A. (1970). The non-muscular phase of the pulmonary circulation in the child. In Proceedings of the Fifth International Cystic Fibrosis Conference, Churchill College, Cambridge, 22/26 Sept. 1969, ed. David Lawson, p. 340. Cystic Fibrosis Research Trust, London.

Leape, L. L., and Longino, L. A. (1964). Infantile lobar emphysema. Pediatrics, 34, 246.

Millard, F. J. C. (1965). The development and the electrocardiographic diagnosis of right ventricular hypertrophy in chronic lung disease. M.D. thesis, University of London.

Reid, L. M. (1967a). The Pathway of Emphysema. Lloyd-Luke, London.

(1967b). The embryology of the lung. In Ciba Foundation Symposium: Development of the I.ung, 1966, p. 109, Edited by Symposium: Development of the I.ung, 1966, p. 109, Edited
de Reuck, A. V. S., and Porter, Ruth. Churchill, London.

- (1970). The laws of lung development. In Proceedings of the Fifth International Cystic Fibrosis Conference, Churchill College, Cambridge, 22/26 Sept. 1969, ed. David Lawson, p. 333. Cystic Fibrosis Research Trust, London.

- Simon, G., Zorab, P. A., and Seidelin, R. (1967). The development of unilateral hypertransradiancy of the lung. Brit. J. Dis. Chest, 61, 190.

Simon, G., and Reid, L. M. (1963). Atresia of an apical bronchus of the left upper lobe-report of three cases. Brit. J. Dis. Chest, 57, 126.

Stovin, P. G. I. (1959). Congenital lobar emphysema. Thorax, 14, 254 Thomson, J., and Forfar, J. O. (1958). Regional obstructive emphysema in infancy. Arch. Dis. Childh., 33, 97.

Waddell, J. A., Simon, G., and Reid, L. (1965). Bronchial atresia of the left upper lobe. Thorax, 20, 214.

Weibel, E. R., and Gomez, D. M. (1962). A principle for counting tissue structures on random sections. J. appl. Physiol., 17, 343. 